\title{
Enterococcal periprosthetic joint infection: clinical and microbiological findings from an 8-year retrospective cohort study
}

\author{
Nora Renz ${ }^{1}$, Rihard Trebse ${ }^{2}$, Doruk Akgün ${ }^{1}$, Carsten Perka ${ }^{1}$ and Andrej Trampuz ${ }^{1 *}$ [D
}

\begin{abstract}
Background: Treatment of enterococcal periprosthetic joint infections (PJI) is challenging due to non-standardized management strategies and lack of biofilm-active antibiotics. The optimal surgical and antimicrobial therapy are unknown. Therefore, we evaluated characteristics and outcome of enterococcal PJI.

Methods: Consecutive patients with enterococcal PJI from two specialized orthopedic institutions were retrospectively analyzed. Both institutions are following the same diagnostic and treatment concepts. The probability of relapse-free survival was estimated using Kaplan-Meier survival curves and compared by log-rank test. Treatment success was defined by absence of relapse or persistence of PJI due to enterococci or death related to enterococcal PJI. Clinical success was defined by the infection-free status, no subsequent surgical intervention for persistent or perioperative infection after re-implantation and no PJl-related death within 3 months.
\end{abstract}

Results: Included were 75 enterococcal PJ episodes, involving 41 hip, 30 knee, 2 elbow and 2 shoulder prostheses. PJ occurred postoperatively in 61 episodes (81\%), hematogenously in $13(17 \%)$ and by contiguous spread in one. $E$. faecalis grew in 64 episodes, E. faecium in 10 and E. casseliflavus in one episode(s). Additional microorganism(s) were isolated in 38 patients (51\%). Enterococci were susceptible to vancomycin in 73 of 75 isolates (97\%), to daptomycin in all 75 isolates, and to fosfomycin in 21 of 22 isolates (96\%). The outcome data was available for 66 patients (88\%). The treatment success after 3 years was $83.7 \%$ (95\% confidence interval [Cl]; 76.1-96.7\%) and the clinical success was 67.5\% (95\% Cl; 57.3-80.8\%). In 11 patients (17\%), a new PJl episode caused by a different pathogen occurred. All failures occurred within 3 years after surgery.

Conclusion: About half of enterococcal PJI were polymicrobial infections. The treatment success was high (84\%). All treatment failures occurred within the first 3 years after revision surgery. Interestingly, 17\% of patients experienced a new PJl caused by another pathogen at a later stage.

Trial registration: The study was retrospectively registered with the public clinical trial identification NCT0253022 at https://www.clinicaltrials.gov on 15 July 2015.

Keywords: Periprosthetic joint infection, Enterococcus spp., Outcome, Microbiology, Biofilm

\footnotetext{
* Correspondence: andrej.trampuz@charite.de

${ }^{1}$ Center for Musculoskeletal Surgery (CMSC), Charité - Universitätsmedizin

Berlin, corporate member of Freie Universität Berlin, Humboldt-Universität zu

Berlin, and Berlin Institute of Health, Charitéplatz 1, 10117 Berlin, Germany

Full list of author information is available at the end of the article
}

(c) The Author(s). 2019 Open Access This article is distributed under the terms of the Creative Commons Attribution 4.0 International License (http://creativecommons.org/licenses/by/4.0/), which permits unrestricted use, distribution, and reproduction in any medium, provided you give appropriate credit to the original author(s) and the source, provide a link to the Creative Commons license, and indicate if changes were made. The Creative Commons Public Domain Dedication waiver (http://creativecommons.org/publicdomain/zero/1.0/) applies to the data made available in this article, unless otherwise stated. 


\section{Background}

Enterococci are reported as causing pathogen of periprosthetic joint infection (PJI) in 2.3 to $15 \%$ [1, $2]$, often as part of a polymicrobial infection [3, 4]. Despite low virulence of the pathogen, the treatment of enterococcal PJI is challenging due to slow bactericidal activity of antimicrobial agents, antimicrobial tolerance and increasing resistance [5]. Data on biofilm-active antibiotics against enterococci are limited or not existing, hence enterococcal PJI were previously classified as "difficult-to-treat" [6]. In addition, some authors report high treatment failure rates [7-9].

Management strategies in enterococcal PJI are controversial. Depending on the surgical procedure applied, divergent outcome results are reported $[1,10-$ 12]. Similarly, antimicrobial treatment recommendations vary widely and predominantly originate from in vitro and experimental studies or are extrapolated from the guidelines for treatment of infective endocarditis. Most authors recommend aminopenicillin derivatives in penicillin-susceptible isolates, whereas vancomycin and linezolid are suggested for penicillinresistant enterococci. Some experts propose single antimicrobial therapy, while others recommend combination treatment with aminoglycosides or ceftriaxone [6,13-15]. Furthermore, the role of rifampin is controversial [16, 17]. Newer antibiotic options such as daptomycin, lipoglycopeptides and fosfomycin are even less investigated for enterococcal PJI [17-19].

We performed a retrospective cohort study in two orthopedic clinics specialized in endoprothetic septic surgery, which are following the same diagnostic and treatment concepts. The aim of the study was to analyze the clinical, laboratory and microbiological characteristics, treatment modalities and outcome of enterococcal PJI.

\section{Methods}

\section{Study design and population}

This retrospective cohort study was conducted in two large orthopedic centers, having a specialized septic surgery unit. PJI episodes caused by Enterococcus species, alone or in combination with another microorganism(s), treated at one of the participating institutions from January 2010 to December 2017 were included. Data on PJI were collected using the same definition criteria, diagnostic procedure and outcome evaluation. The study was approved by the lead ethical committee (approval No. EA04/040/14) and was conducted in accordance with the Declaration of Helsinki. The study was registered with the public clinical trial identification NCT0253022 at https://www.clinicaltrials.gov.

\section{Study definitions}

PJI was defined according to the following definition criteria, as previously used [20-22]. According to these criteria, at least one of the following criteria are needed for the diagnosis of PJI: (i) macroscopic purulence surrounding the prosthesis, (ii) presence of communicating sinus tract, (iii) increased absolute synovial fluid leukocyte count or differential (> 2000 leukocytes/ $\mu$ l or $>$ $70 \%$ granulocytes), (iv) isolation of enterococci from synovial fluid, periprosthetic tissue or sonication culture, (v) positive histopathology, defined as $>23$ granulocytes per 10 high-power fields, corresponding to type II or type III periprosthetic membrane [23]. If enterococci grew in only one microbiological specimen, the microbiological finding was sufficient for the diagnosis of PJI only if additional (non-microbiological) criterion was present, as defined above.

Infections were classified according to their temporal appearance after surgery into early ( $<3$ months), delayed (3-24 months) and late infection ( $>24$ months) [24]. In addition, infections were defined as acute PJI (new onset symptoms for $\leq 4$ weeks), or chronic PJI (symptoms duration $>4$ weeks). The hematogenous route of PJI was defined when (i) the onset of the symptoms was $>3$ months after implantation and occurred after an initial uneventful initial course and (ii) the infection presented with acute onset or the same Enterococcus species grew in blood cultures or from a distant infectious focus. Each case was evaluated and classified by an interdisciplinary team of orthopedic surgeons (DA, RT, CP) and infectious disease specialists (AT, NR).

Infection-free interval describes the interval from primary implantation or last septic surgery of the prosthesis to the diagnosis of an enterococcal infection.

Treatment success was defined by absence of relapse or persistence of PJI due to enterococci or death related to enterococcal PJI.

Clinical success was defined by the presence of all following criteria at last follow-up: (i) infection-free status, characterized by a healed wound without fistula, drainage, and no recurrence of the infection, (ii) no subsequent surgical intervention for persistent or perioperative infection, and (iii) no PJI-related death (within 3 months).

\section{Microbiological testing}

An automated broth microdilution assay was used to determine the antimicrobial susceptibility of all antibiotics except for fosfomycin, and the results were interpreted according to European Committee on Antimicrobial Susceptibility Testing (EUCAST) criteria. For fosfomycin, Etest (bioMérieux, Marcy-l'Étoile, France) was performed in Müller Hinton agar (BD, Heidelberg, Germany) according to manufacturer's instructions. After incubation at 
Table 1 Patient demographics and infection characteristics of 75 patient with enterococcal PJI

\begin{tabular}{|c|c|c|c|c|}
\hline Variable & $\begin{array}{l}\text { All episodes } \\
(n=75)\end{array}$ & $\begin{array}{l}\text { Monomicrobial } \\
(n=37)\end{array}$ & $\begin{array}{l}\text { Polymicrobial } \\
(n=38)\end{array}$ & $P$ value \\
\hline Female sex, No. (\%) & $48(64)$ & $21(57)$ & $27(71)$ & 0.234 \\
\hline Median age (range) - years & $76(30-90)$ & $78(52-90)$ & $75(30-89)$ & 0.810 \\
\hline \multicolumn{5}{|l|}{ Affected joint, No. (\%) } \\
\hline Hip & $41(55)$ & $21(57)$ & $20(53)$ & 0.818 \\
\hline Knee & $30(40)$ & $12(32)$ & $18(47)$ & 0.241 \\
\hline Elbow & $2(3)$ & $2(5)$ & - & 0.240 \\
\hline Shoulder & $2(3)$ & $2(5)$ & - & 0.240 \\
\hline Previous revision surgery, №. (\%) & $61(81)$ & $27(73)$ & $34(89)$ & 0.082 \\
\hline Septic revision & $34(56)$ & $16(59)$ & $18(53)$ & \\
\hline Aseptic revision & $27(44)$ & $11(41)$ & $16(47)$ & \\
\hline
\end{tabular}

Table 2 Infection characteristics of 75 patient with enterococcal PJI

\begin{tabular}{|c|c|c|c|c|}
\hline Variable & $\begin{array}{l}\text { All episodes } \\
(n=75)\end{array}$ & $\begin{array}{l}\text { Monomicrobial } \\
(n=37)\end{array}$ & $\begin{array}{l}\text { Polymicrobial } \\
(n=38)\end{array}$ & $P$ value \\
\hline \multicolumn{5}{|l|}{ Infection manifestation according to the time after last surgery } \\
\hline Early & $27(36)$ & $10(27)$ & $17(45)$ & 0.150 \\
\hline Delayed & $30(40)$ & $17(46)$ & $13(34)$ & 0.351 \\
\hline Late & $18(24)$ & $10(27)$ & $8(21)$ & 0.597 \\
\hline Median delay from last revision to PJI (range) - months & $8.7(0.5-336)$ & $10.2(0.3-336)$ & $5.0(0.4-133)$ & 0.588 \\
\hline Median delay from primary implantation of prosthesis to PJI (range) - months & $47.7(0.3-418)$ & $36.4(0.3-418)$ & $56.6(0.4-373)$ & 0.810 \\
\hline \multicolumn{5}{|l|}{ Presumed route of infection } \\
\hline Perioperative ${ }^{a}$ & $61(81)$ & $25(68)$ & $36(95)$ & 0.003 \\
\hline Hematogenous & $13(17)$ & $12(32)$ & $1(3)$ & $<0.001$ \\
\hline Direct extension by adjacent infectious focus (contiguous) & $1(1)$ & - & $1(3)$ & \\
\hline \multicolumn{5}{|l|}{ Signs and symptoms } \\
\hline Joint pain & $48 / 70(69)$ & $22 / 32(69)$ & 26/38 (68) & 1.000 \\
\hline Local signs & $46(61)$ & $20(54)$ & $26(68)$ & 0.241 \\
\hline Sinus tract & $16(21)$ & $3(8)$ & $13(34)$ & 0.010 \\
\hline Prosthesis loosening in $\mathrm{x}$-ray & $16(21)$ & $5(14)$ & $11(29)$ & 0.158 \\
\hline \multicolumn{5}{|l|}{ Laboratory results at admission } \\
\hline \multicolumn{5}{|l|}{ Serum C-reactive protein } \\
\hline Median (range) - mg/l & $36(1-295)$ & $45(12-229)$ & $36(1-295)$ & 0.936 \\
\hline Increased (> 10 mg/l) & $63(84)$ & $30(81)$ & $33(87)$ & 0.544 \\
\hline \multicolumn{5}{|l|}{ White blood cell count } \\
\hline Median (range) - G/l & $7.9(3.2-34.23)$ & $7.4(4.8-22.4)$ & $8.3(3.2-34.3)$ & 0.992 \\
\hline Increased (> $10 \mathrm{G} / \mathrm{l})$ & $18(24)$ & $8(22)$ & $10(26)$ & 0.788 \\
\hline \multicolumn{5}{|l|}{ Synovial fluid leukocyte count } \\
\hline Increased value ${ }^{b}$ & $25 / 29(86)$ & 16/17 (94) & $9 / 12(75)$ & 0.279 \\
\hline Median absolute count (range) $-10^{3} / \mu \mathrm{l}$ & $20.3(0.42-160)$ & $23(1.8-160)$ & $7.1(0.4-140)$ & 0.097 \\
\hline Histopathology consistent with infection & $53 / 61(87)$ & $26 / 28(93)$ & 27/33 (82) & 0.294 \\
\hline
\end{tabular}

Data are no. (\%) of patients, unless otherwise indicated. Where the denominator is shown, data was not available for all patients

${ }^{a}$ Among them 13 cases (9 polymicrobial and 4 monomicrobial) occurred during surgical and antimicrobial treatment of a PJl caused by another pathogen

${ }^{\mathrm{b}}$ Increased absolute number of leukocytes or percentage of granulocytes 
Table 3 Microbiology of 75 patient with enterococcal PJI

\begin{tabular}{|c|c|c|c|c|}
\hline Variable & $\begin{array}{l}\text { All episodes } \\
(n=75)\end{array}$ & $\begin{array}{l}\text { Monomicrobial } \\
(n=37)\end{array}$ & $\begin{array}{l}\text { Polymicrobial } \\
(n=38)\end{array}$ & $P$ value \\
\hline \multicolumn{5}{|l|}{ Enterococcus spp. } \\
\hline E. faecalis & $64(85)$ & $33(89)$ & $31(82)$ & 0.516 \\
\hline E. faecium ${ }^{a}$ & $10(13)$ & $4(11)$ & $6(16)$ & 0.736 \\
\hline E. casseliflavus & $1(1)$ & - & $1(3)$ & 1.000 \\
\hline \multicolumn{5}{|l|}{ Co-pathogens in mixed infections } \\
\hline Coagulase-negative staphylococci ${ }^{\mathrm{b}}$ & & & 20 & \\
\hline Gram-negative bacillic ${ }^{c}$ & & & 18 & \\
\hline Anaerobes $^{d}$ & & & 5 & \\
\hline Candida sppe & & & 4 & \\
\hline Streptococcus spp ${ }^{f}$ & & & 3 & \\
\hline Staphylococcus aureus & & & 2 & \\
\hline Corynebacterium spp ${ }^{g}$ & & & 2 & \\
\hline \multicolumn{5}{|l|}{ Source of pathogen isolation } \\
\hline Synovial fluid & $42 / 55(76)$ & $20 / 25(80)$ & $22 / 30(73)$ & 0.752 \\
\hline Periprosthetic tissue & $52 / 67(78)$ & 24/33 (73) & 28/34 (82) & 0.392 \\
\hline Sonication fluid & $33 / 45(73)$ & $15 / 22(68)$ & $18 / 23(78)$ & 0.514 \\
\hline
\end{tabular}

Data are no. (\%) of patients, unless otherwise indicated. Where the denominator is shown, data was not available for all patients

ancluding 2 vancomycin-resistant enterococci (VRE)

${ }^{\mathrm{b}}$ S. epidermidis $(n=14)$, S. capitis $(n=2)$, S. lugdunensis $(n=2)$, S. saccharolyticus $(n=1)$ and S. haemolyticus $(n=1)$

${ }^{c}$ Klebsiella spp. $(n=6)$, E. coli $(n=5)$, Proteus spp. $(n=5)$, Pseudomonas aeruginosa $(n=4)$, Morganella spp. $(n=2)$, Serratia marcescens $(n=1)$, Enterobacter cloacae

$(n=1)$, Acinetobacter spp. $(n=1)$

${ }^{\mathrm{d} F i n e g o l d i a}$ magna $(n=3)$, Peptinophilus asaccharolyticus $(n=1)$, Peptostreptococcus micros $(n=1)$, Bacteroides fragilis $(n=1)$

${ }^{\mathrm{e}} \mathrm{C}$. albicans $(n=4)$, C. parapsilosis $(n=1)$

${ }^{\text {f }}$. salivarius $(n=1)$, S. cristatus $(n=1)$, not further specified $(n=1)$

${ }^{g}$ C. striatum $(n=1), C$. tuberculostearicum $(n=1)$

$37^{\circ} \mathrm{C}$ for $24 \mathrm{~h}$, the minimal inhibitory concentration (MIC) was recorded as the concentration value where the inhibition ellipse intersected the scale of the strip.

\section{Surgical treatment}

All patients underwent revision surgery. Patients with acute (early postoperative or late hematogenous) infection with symptoms lasting $<4$ weeks were treated with retention of the prosthesis, change of the mobile parts and meticulous debridement. In contrast, patients with chronic PJI, with signs of infection lasting $\geq 4$ weeks or with a loose prosthesis were treated with one-stage or two-stage revision, depending on the local soft tissue and bone conditions and the revision history.

\section{Antimicrobial treatment}

Empiric antibiotic treatment was started intravenously after surgery and was subsequently adapted according to the susceptibility of the isolated organism. The intravenous treatment was typically continued for at least 2 weeks, followed by oral antibiotic treatment, as previously described $[25,26]$. In case of a two-stage revision, antibiotics were administered without interruption until re-implantation. After re-implantation, antibiotics were continued to complete a total duration of at least 12 weeks (or longer, if the prosthesis-free interval was $>6$ weeks).

Adequate antimicrobial therapy was considered when the antibiotic was appropriate against enterococcal infection according to its activity, dose, oral bioavailability and bone penetration. The antibiotics were chosen according to institutional treatment guidelines and patient history of antibiotic allergies. The following intravenous doses were used in patients with normal renal function: vancomycin $15-20 \mathrm{mg} / \mathrm{kg}$ every $12 \mathrm{~h}$, daptomycin $8-10$ $\mathrm{mg} / \mathrm{kg}$ once daily, fosfomycin $5 \mathrm{~g}$ every $8 \mathrm{~h}$, penicillin $\mathrm{G}$ 5 million IU every $6 \mathrm{~h}$, ampicillin $2 \mathrm{~g}$ every $6 \mathrm{~h}$, gentamicin $3 \mathrm{mg} / \mathrm{kg}$ once daily. In case of concomitant infective endocarditis, higher doses were administered according to the respective guidelines.

\section{Follow-up evaluation}

Patients were scheduled for follow-up in the outpatient clinic at 3, 6, 12 and 24 months after revision surgery. Clinical, laboratory and radiological evaluation was performed and interpreted interdisciplinary by an orthopedic surgeon and an infectious disease specialist. Further follow-up evaluation was performed by phone contact using a standardized case-report form. 


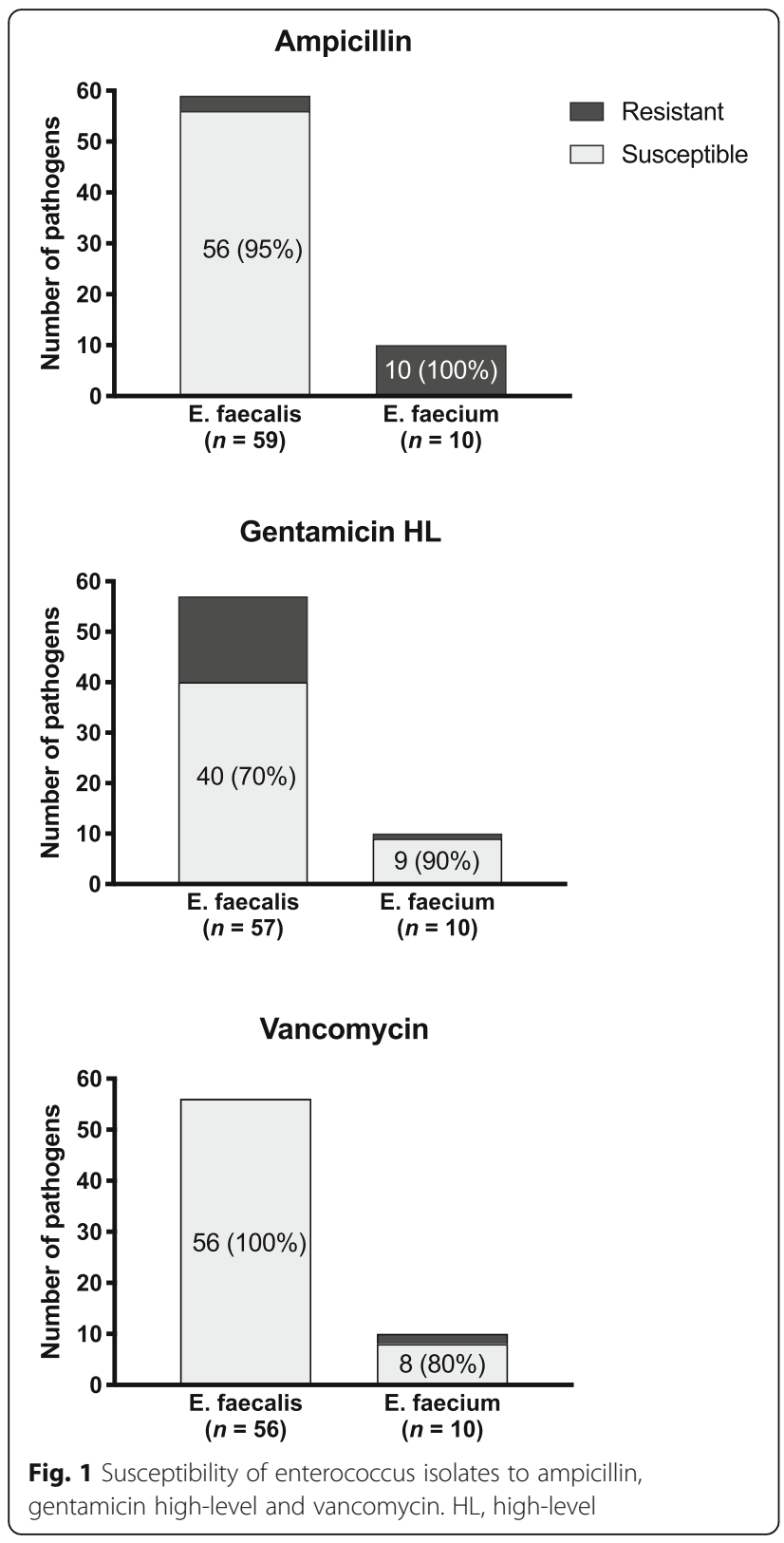

\section{Statistical analysis}

Categorical variables were compared using the Fisher's exact test, for comparison of continuous variables the Mann-Whitney $\mathrm{U}$ test was applied. A two-sided $p$ value of $<0.05$ was considered significant. The probability of infection-free survival and 95\% confidence interval (CI) was estimated using the Kaplan-Meier survival method. Outcomes between groups were compared using Fishers exact test. An alpha level of 0.05 was considered significant. For statistical analysis and graphics, Prism software (version 8.2; GraphPad, La Jolla, CA, USA) was used.

\section{Results}

Patient demographical data

A total of 75 patients were included. The demographics of 37 monomicrobial and 38 polymicrobial enterococcal PJI are shown in Table 1. At least one previous revision surgery was performed in 61 patients $(81 \%)$, the number of previous surgeries ranged from 1 to 18 interventions.

\section{Infection characteristics (Table 2)}

Twenty-seven episodes (36\%) occurred early after surgery, 30 (40\%) delayed and 18 (24\%) late.

The presumed route of infection was perioperative in the majority of PJI (81\%), whereas the hematogenous route was documented in 13 episodes (17\%). The primary infection focus was found in 7 cases, including infective endocarditis in four patients and urinary tract infection in three patients. In 13 patients, enterococci were detected as second pathogen, isolated during treatment of PJI initially caused by another pathogen.

Enterococcal PJI presented with acute onset in 33 patients (44\%) and with chronic symptoms in 42 (56\%). Sinus tract was more often observed in polymicrobial than monomicrobial infections (34\% vs. $8 \%, p=0.010)$.

Among diagnostic tests, positivity rate of histopathology of periprosthetic tissue (87\%), synovial fluid leukocyte count $(86 \%)$ and serum C-reactive protein concentration (84\%) were high, whereas only 18 patients $(24 \%)$ presented with elevated serum white blood cell count.

\section{Microbiological findings}

Table 3 summarizes the isolated enterococcal species and source of pathogen isolation. PJI was caused by E. faecalis in 64 patients $(85 \%)$, by E. faecium in 10 patients $(13 \%)$ and by $E$. casseliflavus in 1 patient (1\%). The most common co-pathogens in polymicrobial infections were coagulase-negative staphylococci and gram-negative bacilli.

Figure 1 summarizes the antimicrobial susceptibilities of enterococci to ampicillin, high-level of gentamicin and vancomycin. Of 22 enterococcal isolates, $21 \mathrm{had}$ an MIC for fosfomycin of $\leq 128 \mathrm{mg} / \mathrm{l}$ (Fig. 2).

In 13 patients, only one specimen (tissue in 4 patients and synovial fluid in 9 patients) grew the Enterococcus spp. In all of them, a non-microbiological criterion was fulfilled, i.e. among positive histopathology in 9 patients, purulent secretion in 3 patients and positive synovial fluid leukocyte count in 1 patient.

\section{Surgical treatment}

Table 4 summarizes the surgical and antimicrobial treatment of analyzed patients. Retention of the prosthesis was performed in 13 patients with acute infection, onestage exchange in 10 patients ( 2 acute and 8 chronic PJI) 


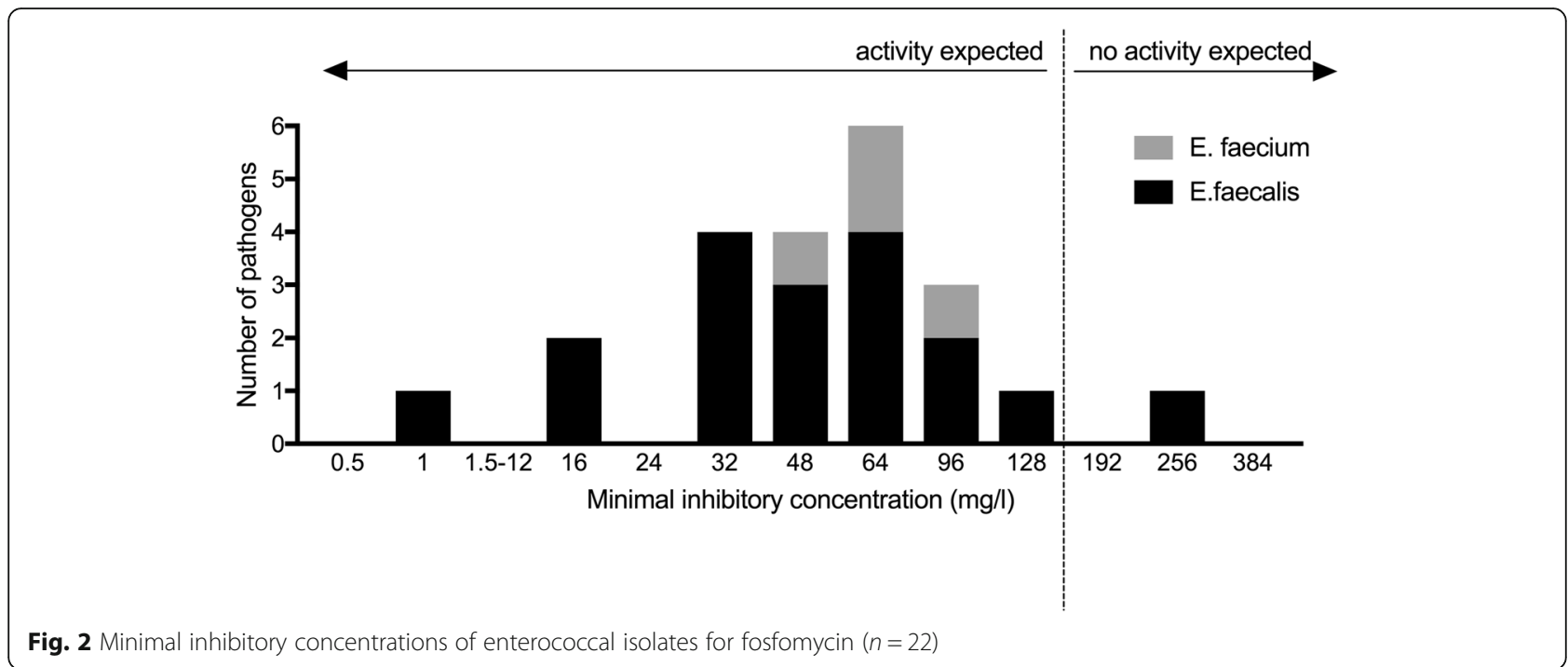

and two-stage exchange in 43 patients. The median interval between explantation and re-implantation surgery was 84 days (range, 29-292 days). In 9 patients (4 hips, 4 knees, 1 elbow) resection arthroplasty was performed.

\section{Antimicrobial treatment}

Antimicrobial therapy was administered in 74 patients (99\%), all of them were initially treated with intravenous antibiotics. Fifty-nine patients $(80 \%)$ received a combination therapy with the addition of gentamicin $(n=24)$,

Table 4 Surgical and antimicrobial treatment of 75 patient with enterococcal PJI

\begin{tabular}{|c|c|c|c|c|}
\hline & $\begin{array}{l}\text { All episodes } \\
(n=75)\end{array}$ & $\begin{array}{l}\text { Mono-microbial } \\
(n=37)\end{array}$ & $\begin{array}{l}\text { Poly-microbial } \\
(n=38)\end{array}$ & $P$ value \\
\hline \multicolumn{5}{|l|}{ Surgical treatment } \\
\hline Prosthesis retention ${ }^{a}$ & $13(17)$ & 7 & 6 & 0.768 \\
\hline One-stage exchange ${ }^{b}$ & $10(13)$ & 5 & 5 & 1.000 \\
\hline Multi-stage exchange $^{c}$ & $43(57)$ & 20 & 23 & 0.644 \\
\hline No. surgical interventions needed, median (range) & $2(2-8)$ & $2(2-5)$ & $3(2-8)$ & 0.039 \\
\hline Resection arthroplasty & $9(12)$ & 5 & 4 & 0.736 \\
\hline \multicolumn{5}{|l|}{ Antimicrobial treatment $^{\mathrm{d}}$} \\
\hline \multicolumn{5}{|l|}{ Intravenous antibiotic agent } \\
\hline Penicillin derivative & $61 / 74(82)$ & $30 / 37(81)$ & $31 / 37(84)$ & 1.000 \\
\hline Vancomycin or daptomycin & $12 / 74(16)$ & $6 / 37(16)$ & $6 / 37(16)$ & 1.000 \\
\hline Other & $1 / 74(3)$ & $1 / 37(3)$ & - & \\
\hline \multicolumn{5}{|l|}{ Additive agent for combination treatment } \\
\hline Fosfomycin & 17/74 (23) & 9/37 (24) & $8 / 37(22)$ & 1.000 \\
\hline Gentamicin & $16 / 74(22)$ & $11 / 37(30)$ & $5 / 37(14)$ & 0.157 \\
\hline Fosfomycin and gentamicin & $8 / 74(11)$ & $5 / 37(14)$ & 3/37 (8) & 0.711 \\
\hline Vancomycin or daptomycin & 18/74 (24) & $4 / 37(11)$ & 14/37 (38) & 0.013 \\
\hline None & 15/74 (20) & $8 / 37(22)$ & $7 / 37(20)$ & 1.000 \\
\hline Median total duration (range) - weeks $^{d}$ & $16(2-52)$ & $15(4-39)$ & $16(2-52)$ & 0.459 \\
\hline Prolonged treatment duration (> 12 weeks) & $35 / 74(47)$ & $17 / 37(46)$ & 18/37 (49) & 1.000 \\
\hline
\end{tabular}

Data are no. (\%) of patients, unless otherwise indicated. Where the denominator is shown, data was not available for all patients

ancluding exchange of mobile parts $(n=7)$, debridement only $(n=4)$ and no surgery $(n=1)$

${ }^{b}$ In 3 patients, only partial exchange was performed

${ }^{\mathrm{C}}$ Median interval between explantation and re-implantation was 84 days (range, 29-292 days)

${ }^{\mathrm{d} O n e}$ patient received no antimicrobial treatment 
Table 5 Clinical outcome of 75 patient with enterococcal PJI

\begin{tabular}{|c|c|c|c|c|}
\hline Outcome & $\begin{array}{l}\text { All episodes } \\
(n=66)\end{array}$ & $\begin{array}{l}\text { Monomicrobial } \\
(n=33)\end{array}$ & $\begin{array}{l}\text { Polymicrobial } \\
(n=33)\end{array}$ & $P$ value \\
\hline Median follow-up (range) - months & $31.8(0.3-83.8)$ & $25.9(1.4-78.6)$ & $33.8(0.3-83.8)$ & 0.138 \\
\hline Clinical success & $45(68)$ & $22(66)$ & $23(70)$ & 1.000 \\
\hline Clinical failure $^{a}$ & $21(31)$ & $11(33)$ & $10(30)$ & \\
\hline Isolation of another or no pathogen & 11 & 5 & 6 & \\
\hline Treatment failure $^{b}$ & 10 & 6 & 4 & \\
\hline Treatment success & $56(85)$ & $27(82)$ & $29(88)$ & 0.752 \\
\hline
\end{tabular}

Data are no. (\%) of patients, unless otherwise indicated. Where the denominator is shown, data was not available for all patients

${ }^{a} 6$ patients died, 2 death were associated with the enterococcal PJI (treatment failure), 1 death was related to a new infection caused by staphylococci in the later course (clinical failure) and 3 patients died from a non-infectious cause ( 1 tumor, 2 pulmonary embolism) and were considered infection-free at the time of death

bersistent infection or relapse with same Enterococcus spp. as initially isolated

vancomycin or daptomycin $(n=18)$ or fosfomycin $(n=$ 25). The median duration of fosfomycin treatment was 14 days (range, 3-90 days).

The antibiotic treatment was considered adequate in 66 patients (88\%), which included either penicillin derivative (in ampicillin-susceptible enterococci) or vancomycin or daptomycin (in ampicillin-resistant enterococci). The median duration of antibiotic treatment was 16 weeks (range, 2-52 weeks).

\section{Outcome evaluation}

Table 5 shows outcome data of 66 patients (88\%) for whom the follow-up data was available and who completed antibiotic therapy. The median follow-up was 31.8 months (range, $0.3-83.3$ months). The relapse-free probability (treatment success) after 3 years was 83.7 (95\% CI; 76.1-96.7\%) and the infection-free probability (clinical success) was 67.5\% (95\% CI; 57.3-80.8\%) (Fig. 3). All failures occurred within 3 years after diagnosis of enterococcal PJI. Among 21 patients with clinical failure, 10 experienced persistent infection or relapse due to enterococci (treatment failure) and 11 experienced new infection with another microorganism or culture-negative (nine of which occurred within 12 months). Two patients died within the first 3 months after surgical revision related to infection. Of 21 patients with clinical failure, 18 (86\%) had previous revision surgery.

Clinical and treatment outcome according to surgical procedures is summarized in Fig. 4. Polymicrobial and monomicrobial infections showed no difference regarding treatment outcome ( $88 \%$ vs. $82 \%, p=0.733)$. Adequate antimicrobial treatment was associated with better treatment outcome compared to inadequate therapy (91\% vs. $38 \%, p=0.002)$. No statistical difference was observed in the treatment outcome in patients receiving antimicrobial monotherapy or combination therapy $(88 \%$ vs. $73 \%, p=$ $0.217)$, neither in those receiving fosfomycin in their treatment regimen (95\%) compared to those without fosfomycin $(80 \%)(p=0.261)$. Prolonged antimicrobial therapy of
$>12$ weeks was also not associated with better treatment outcome than shorter therapy ( $81 \%$ vs. $85 \%$, respectively, $p=0.920)$.

\section{Discussion}

Outcome reports of enterococcal PJI vary widely, to a certain extent due to heterogeneous definition of treatment success. While some authors defined failure as relapse or persistent infection with isolation of the same pathogen, others also considered new infections caused by other pathogens as failures. In our study, failures were subclassified as treatment or clinical failure and success, respectively. Considering these definitions, the eradication of enterococcal PJI (treatment success) in our cohort was $83.7 \%$, which was higher than previously reported (50 to $76 \%$ ) [1, 9, 12, 27]. However, as 11 patients experienced new infection with another microorganism or were culture-negative, the infection-free probability (clinical success) was only $67.5 \%$.

Rasouli et al. [1] described clinical success in 32 of 36 patients $(89 \%)$ and treatment success in 35 of 36 patients (97\%) with enterococcal PJI. However, in several patients additional major surgeries were performed such as resection arthroplasty, two-stage exchange, above-knee amputation or fusion. The fact that in our study no new PJIs occurred in patients with prosthesis retention and only in one patient undergoing one-stage exchange, corroborates the assumption that multiple surgeries may be a risk factor for introduction of a new pathogen. It remains unclear whether these infections are really "new" or a relapse of an unrecognized initial pathogen. Whereas adequate antimicrobial treatment was associated with better treatment outcome, prolonged antimicrobial therapy of $>12$ weeks did not influence the outcome.

The EUCAST recommendations do not provide breakpoints for enterococci and fosfomycin and, thus, this substance is not recommended as monotherapy for the treatment of enterococcal infections. However, in vitro 


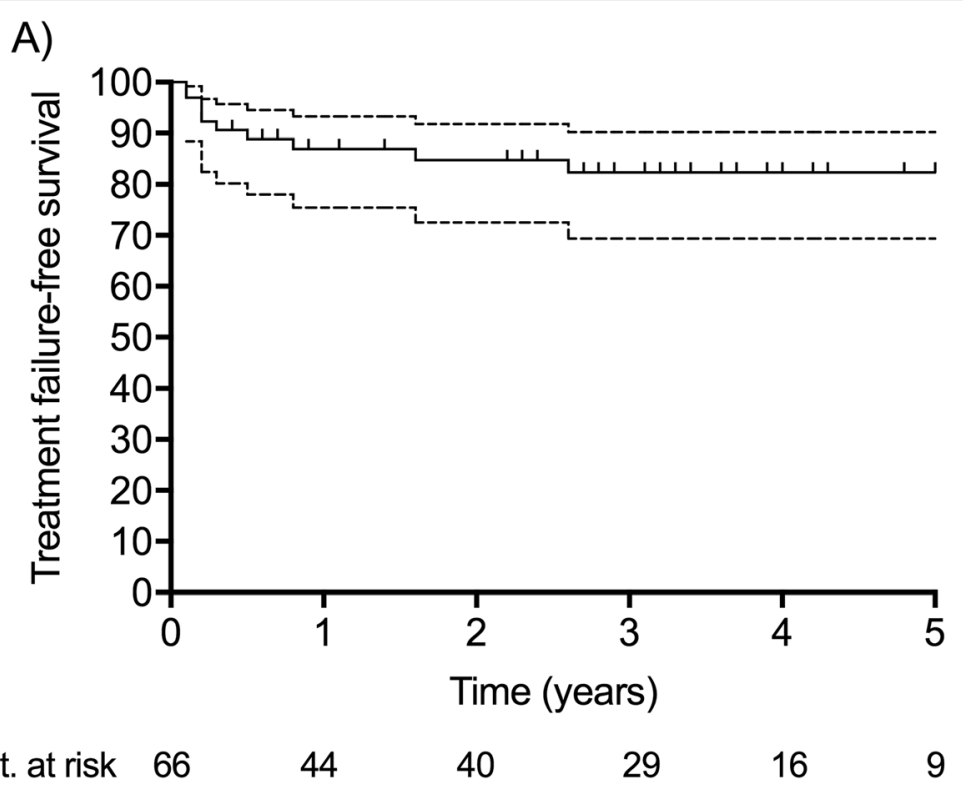

B)

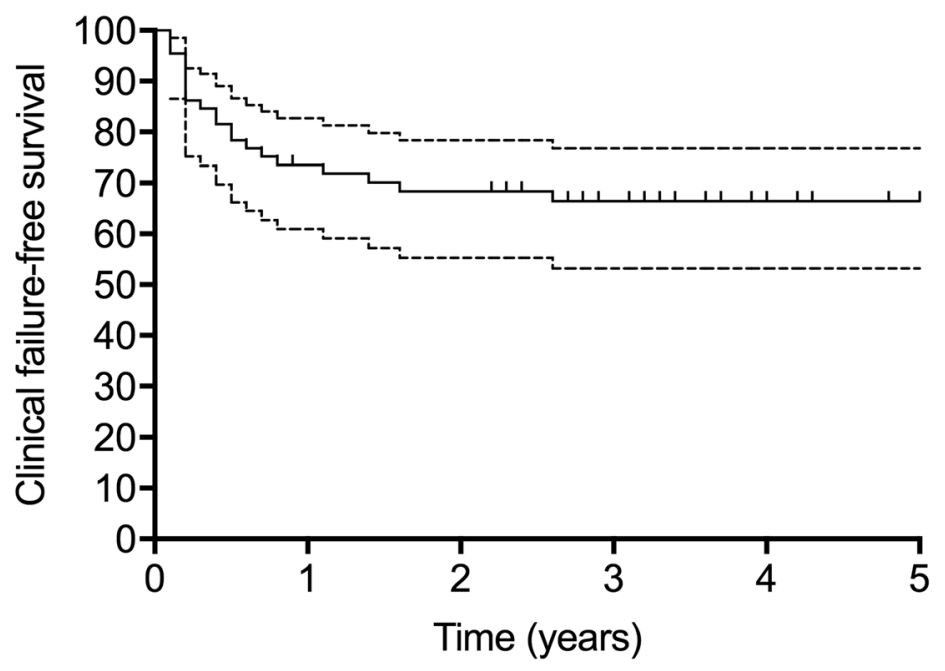

$\begin{array}{llllll}\text { Pat at risk } 66 & 44 & 40 & 29 & 16 & 9\end{array}$

Fig. 3 Outcome analysis of 75 patient with enterococcal PJ. Treatment failure (a) and clinical failure (b). Dotted lines indicate the $95 \%$ confidence interval

and animal model data showed activity of fosfomycin against planktonic and adherent $E$. faecalis. The most efficient regimen for killing planktonic and adherent $E$. faecalis was the combination of fosfomycin and gentamicin [17]. Extrapolated from the recommendation for the use of fosfomycin as combination partner for the treatment of infections caused by wild type isolates of Pseudomonas spp. with an epidemiological cut-off of $128 \mathrm{mg} / \mathrm{l}$ [28], the same cut-off is also used for enterococci and intravenous fosfomycin in daily routine. In our cohort, fosfomycin was active against most enterococcal isolates using the aforementioned fosfomycin MIC cutoff value. When intravenous fosfomycin $(5 \mathrm{~g}$ every $8 \mathrm{~h}$ intravenously) was included in the treatment regimen, the treatment outcome was numerically higher than without intravenous fosfomycin ( $95 \%$ vs. $80 \%)$, however, the difference did not reach statistical significance. Prospective randomized controlled trials are needed to further explore the role of fosfomycin in biofilm-related infections.

Debridement and prosthesis retention was associated with the highest treatment success; however, this 


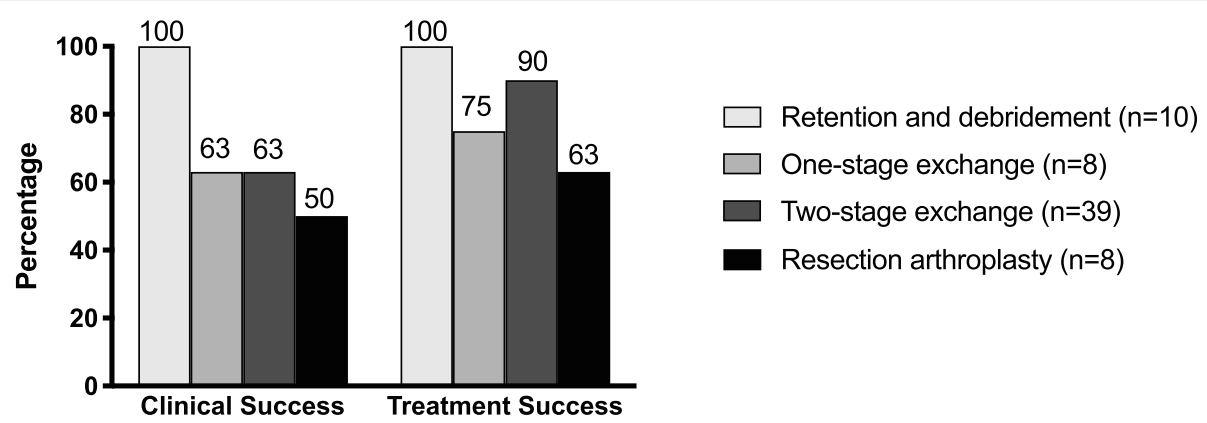

Fig. 4 Clinical and treatment success depending on the performed surgical procedure in 66 patients with enterococcal PJI with available follow up. One patient in whom no surgery was performed experienced treatment failure

observation is most likely biased by rather treating "uncomplicated" and acute infections with retention and more complex ones with prosthesis removal. This fact represents the main limitation of the study. Other studies reported low clinical success with debridement and retention such as 40\% [9], 47\% [27], 50\% [1] and 66\% [11] and based on this observation two-stage prosthesis exchange is recommended for enterococcal PJI. However, with improved antimicrobial therapy, debridement and retention or one-stage prosthesis exchange may become an alternative to two-stage exchange with high treatment success. Based on our data, in our institution PJI caused by enterococci are no longer managed as difficult-to-treat infections as previously suggested [6]. Of note, permanent removal of prosthesis (resection arthroplasty without reimplantation) was performed in in 9 patients (12\%) in our cohort. These patients were cured from infection, although the result may represent a functional inferior outcome, if they were able to walk before occurrence of PJI. Reasons for choosing resection arthroplasty were high risk of complications due to additional surgery, exceptionally insufficient bone stock or bedriddeness of patients.

In a previous study, the clinical presentation of enterococcal PJI was similar to that caused by low-virulent organisms such as Cutibacterium acnes and coagulasenegative staphylococci [12]. Accordingly, it remains unclear whether enterococci are true pathogens or contaminants when grown in a single specimen. If isolated in a single specimen, typical members of the normal skin flora are considered contaminants. As enterococci are not typically colonizing the skin, contamination during sampling appears unlikely. A similar question was investigated by Jindai et al. for positive blood cultures for enterococci [29]. The authors concluded that single-positive blood cultures likely indicate true infection rather than contamination, as the clinical outcome of patients with singlepositive blood cultures was similar to those with multiplepositive blood cultures. In our study at least two positive Enterococcus spp. cultures were required, as defined by other authors evaluating enterococcal PJI [1, 9, 12]. However, a single positive culture was accepted if an additional non-microbiological criterion was present.

All treatment failures in our cohort occurred within the first 3 years after treatment of enterococcal PJI. Based on this finding, we recommend close follow-up in the first years, whereas the likelihood of relapse afterwards seems low. The risk of hematogenous PJI, however, remains during the whole indwelling time of the prosthesis, as recently demonstrated [30].

\section{Conclusions}

Although the retrospective design of the study and the limited number of patients make definitive conclusions regarding outcome difficult, some important finding can be deduced from the presented study. First, about half of enterococcal PJI were polymicrobial infections. Second, the treatment success was unexpectedly high $(84 \%)$, suggesting that enterococcal PJI are not difficult to treat. All treatment failures occurred within the first 3 years after revision surgery. Third, $17 \%$ of patients experienced a new PJI caused by another pathogen at a later stage. Fourth, intravenous fosfomycin was active against 21 of 22 enterococcal isolates (MIC $\leq 128 \mu \mathrm{g} / \mathrm{ml}$ ) and its value should be further explored in prospective clinical trials involving enterococcal PJI.

\section{Abbreviations}

Cl: Confidence interval; EUCAST: European Committee on Antimicrobial Susceptibility Testing; MIC: Minimal inhibitory concentration;

PJl: Periprosthetic joint infections

\section{Acknowledgements \\ None.}

\section{Authors' contributions}

NR collected, analyzed and interpreted the patient data and was the major contributor in writing the manuscript draft. RT participated in the stud design, contributed the patient data and revised the manuscript draft. DA and CP participated in the study design, patient data collection and revised the manuscript. AT designed the study design, interpreted the patient data and revised the manuscript. All authors read and approved the final manuscript. 


\section{Funding}

This work was supported by the PRO-IMPLANT Foundation, Berlin, Germany (https://www.pro-implant-foundation.org) providing an unrestricted research grant. We acknowledge also support from the German Research Foundation (DFG) and the Open Access Publication Fund of Charité - Universitätsmedizin Berlin, Berlin, Germany. The funding bodies had no influence on the design of the study, collection, analysis, and interpretation of data and in writing the manuscript.

\section{Availability of data and materials}

The datasets used and/or analysed during the current study are available from the corresponding author on reasonable request.

\section{Ethics approval and consent to participate}

The study was approved by the lead ethical committee (approval No. EA04/ 040/14 at Charité - Universitätsmedizin Berlin, Berlin, Germany) and was registered with the public clinical trial identification NCT0253022 at https://www.clinicaltrials.gov. The need for patient informed consent was waived by the ethical committee for this retrospective study.

\section{Consent for publication}

Not applicable.

\section{Competing interests}

The authors declare that they have no competing interests.

\section{Author details}

${ }^{1}$ Center for Musculoskeletal Surgery (CMSC), Charité - Universitätsmedizin Berlin, corporate member of Freie Universität Berlin, Humboldt-Universität zu Berlin, and Berlin Institute of Health, Charitéplatz 1, 10117 Berlin, Germany. ${ }^{2}$ Orthopaedic Hospital Valdoltra, SI-6280 Ankaran, Slovenia.

\section{Received: 4 August 2019 Accepted: 8 December 2019}

\section{Published online: 27 December 2019}

\section{References}

1. Rasouli MR, Tripathi MS, Kenyon R, Wetters N, Della Valle CJ, Parvizi J. Low rate of infection control in enterococcal periprosthetic joint infections. Clin Orthop Relat Res. 2012;470(10):2708-16.

2. Fernandes A, Dias M. The microbiological profiles of infected prosthetic implants with an emphasis on the organisms which form biofilms. J Clin Diagn Res. 2013;7(2):219-23.

3. Peel TN, Buising KL, Dowsey MM, Aboltins CA, Daffy JR, Stanley PA, Choong PF. Outcome of debridement and retention in prosthetic joint infections by methicillin-resistant staphylococci, with special reference to rifampin and fusidic acid combination therapy. Antimicrob Agents Chemother. 2013;57(1): 350-5.

4. Cobo J, Miguel LG, Euba G, Rodriquez D, Garcia-Lechuz JM, Riera M, Falgueras L, Palomino J, Benito N, del Toro MD, et al. Early prosthetic joint infection: outcomes with debridement and implant retention followed by antibiotic therapy. Clin Microbiol Infect. 2011;17(11):1632-7.

5. Murray BE. The life and times of the Enterococcus. Clin Microbiol Rev, 1990;3(1):46-65.

6. Zimmerli W, Trampuz A, Ochsner PE. Prosthetic-joint infections. N Engl J Med. 2004;351(16):1645-54

7. Soriano A, Garcia S, Bori G, Almela M, Gallart X, Macule F, Sierra J, Martinez JA, Suso S, Mensa J. Treatment of acute post-surgical infection of joint arthroplasty. Clin Microbiol Infect. 2006;12(9):930-3.

8. Lora-Tamayo J, Euba G, Ribera A, Murillo O, Pedrero S, García-Somoza D, Pujol M, Cabo X, Ariza J. Infected hip hemiarthroplasties and total hip arthroplasties: Differential findings and prognosis. J Infect. 2013;67(6):536-44.

9. Kheir MM, Tan TL, Higuera C, George J, Della Valle CJ, Shen M, Parvizi J. Periprosthetic joint infections caused by enterococci have poor outcomes. J Arthroplast. 2017;32(3):933-47.

10. Tornero E, Senneville E, Euba G, Petersdorf S, Rodriguez-Pardo D, Lakatos B, Ferrari MC, Pilares M, Bahamonde A, Trebse R, et al. Characteristics of prosthetic joint infections due to Enterococcus sp. and predictors of failure: a multi-national study. Clin Microbiol Infect. 2014;20(11):1219-24.

11. Duijf SV, Vos FJ, Meis JF, Goosen JH. Debridement, antibiotics and implant retention in early postoperative infection with Enterococcus sp. Clin Microbiol Infect. 2015;21(5):e41-2.
12. El Helou OC, Berbari EF, Marculescu CE, El Atrouni WI, Razonable RR, Steckelberg JM, Hanssen AD, Osmon DR. Outcome of enterococcal prosthetic joint infection: is combination systemic therapy superior to monotherapy? Clin Infect Dis. 2008;47(7):903-9.

13. Osmon DR, Berbari EF, Berendt AR, Lew D, Zimmerli W, Steckelberg JM, Rao N, Hanssen A, Wilson WR. Infectious diseases Society of a: diagnosis and management of prosthetic joint infection: clinical practice guidelines by the Infectious Diseases Society of America. Clin Infect Dis. 2013;56(1):e1-e25.

14. Tande AJ, Patel R. Prosthetic joint infection. Clin Microbiol Rev. 2014;27(2):302-45.

15. Euba G, Lora-Tamayo J, Murillo O, Pedrero S, Cabo J, Verdaguer R, Ariza J. Pilot study of ampicillin-ceftriaxone combination for treatment of orthopedic infections due to Enterococcus faecalis. Antimicrob Agents Chemother. 2009;53(10):4305-10

16. Holmberg A, Morgelin M, Rasmussen M. Effectiveness of ciprofloxacin or linezolid in combination with rifampicin against Enterococcus faecalis in biofilms. J Antimicrob Chemother. 2012;67(2):433-9.

17. Oliva A, Furustrand Tafin U, Maiolo EM, Jeddari S, Betrisey B, Trampuz A. Activities of fosfomycin and rifampin on planktonic and adherent Enterococcus faecalis strains in an experimental foreign-body infection model. Antimicrob Agents Chemother. 2014;58(3):1284-93.

18. Descourouez $J$, Jorgenson MR, Wergin JE, Rose WE. Fosfomycin synergy in vitro with amoxicillin, daptomycin, and linezolid against vancomycinresistant Enterococcus faecium from renal transplant patients with infected urinary stents. Antimicrob Agents Chemother. 2013;57(3):1518-20.

19. Yuste JR, Quesada M, Diaz-Rada P, Del Pozo JL. Daptomycin in the treatment of prosthetic joint infection by Enterococcus faecalis: safety and efficacy of high-dose and prolonged therapy. Int J Infect Dis. 2014;27:65-6.

20. Renz N, Yermak K, Perka C, Trampuz A. Alpha defensin lateral flow test for diagnosis of periprosthetic joint infection. Not a screening but a confirmatory test. J Bone Joint Surg Am. 2018;100(9):742-50.

21. Zimmerli W. Clinical presentation and treatment of orthopaedic implantassociated infection. J Intern Med. 2014;276(2):111-9.

22. Sigmund IK, Yermak K, Perka C, Trampuz A, Renz N. Is the enzyme-linked Immunosorbent assay more accurate than the lateral flow alpha Defensin test for diagnosing Periprosthetic joint infection? Clin Orthop Relat Res. 2018;476(8):1645-54

23. Krenn V, Morawietz L, Perino G, Kienapfel H, Ascherl R, Hassenpflug GJ, Thomsen M, Thomas P, Huber M, Kendoff D, et al. Revised histopathological consensus classification of joint implant related pathology. Pathol Res Pract. 2014;210(12):779-86.

24. Trampuz A, Piper KE, Jacobson MJ, Hanssen AD, Unni KK, Osmon DR, Mandrekar JN, Cockerill FR, Steckelberg JM, Greenleaf JF, et al. Sonication of removed hip and knee prostheses for diagnosis of infection. N Engl J Med. 2007;357(7):654-63.

25. Izakovicova P, Borens O, Trampuz A. Periprosthetic joint infection: current concepts and outlook. EFORT Open Rev. 2019;4(7):482-94.

26. Winkler T, Stuhlert MGW, Lieb E, Muller M, von Roth P, Preininger B, Trampuz A, Perka CF. Outcome of short versus long interval in two-stage exchange for periprosthetic joint infection: a prospective cohort study. Arch Orthop Trauma Surg. 2019;139(3):295-303.

27. Tornero E, Soriano A. Prosthetic joint infection due to Enterococcus sp treated with debridement, antibiotics and retention of the implant (DAIR). Clin Microbiol Infect. 2015;21(5):e43-4.

28. The European Committee on Antimicrobial Susceptibility Testing (EUCAST) (2018) Breakpoint tables for interpretation of MICs and zone diameters. version 8.0 2018, http://www.eucast.org/clinical_breakpoints.

29. Jindai K, Strerath MS, Hess T, Safdar N. Is a single positive blood culture for Enterococcus species representative of infection or contamination? Eur J Clin Microbiol Infect Dis. 2014;33(11):1995-2003.

30. Rakow A, Perka C, Trampuz A, Renz N. Origin and characteristics of haematogenous periprosthetic joint infection. Clin Microbiol Infect. 2018;25: $845-50$.

\section{Publisher's Note}

Springer Nature remains neutral with regard to jurisdictional claims in published maps and institutional affiliations. 